\title{
Analytic and Clustering Properties of Thermodynamic Functions and Distribution Functions for Classical Lattice and Continuum Systems*
}

\author{
J. L. Lebowitz and O. Penrose \\ Belfer Graduate School of Science, Yeshiva University, New York
}

Received June 1, 1968

\begin{abstract}
Our most complete results concern the Ising spin system with purely ferromagnetic interactions in a magnetic field $H$ (or the corresponding lattice gas model with fugacity $z=$ const. $\exp (-2 m H \beta)$ where $m$ is the magnetic moment of each spin). We show that, in the limit of an infinite lattice, (i) the free energy per site and the distribution functions $n_{s}\left(\boldsymbol{x}_{1}, \ldots, \boldsymbol{x}_{s} ; \beta, z\right)$ are analytic in the two variables $\beta$ and $H$ if the reciprocal temperature $\beta>0$ and the complex number $H$ is not a limit point of zeros of the grand partition function $\Xi$, and (ii) the Ursell functions $u_{s}\left(\boldsymbol{x}_{1}, \ldots, \boldsymbol{x}_{s} ; \beta, z\right)$ tend to 0 as $\Delta_{s} \equiv \max _{i, j}\left|\boldsymbol{x}_{i}-\boldsymbol{x}_{j}\right| \rightarrow \infty$ if $\beta>0$ and $\operatorname{Re} H \neq 0$; in particular, if the interaction potential vanishes for separations exceeding some fixed cutoff value $\lambda$, then $\left|u_{s}\right|<C \exp \left[(-2 \beta m|\operatorname{Re} H|+\varepsilon) \Delta_{s} \mid \lambda\right]$ where $\varepsilon$ is any small positive number and $C$ is independent of $\Delta_{s}$. One consequence of the result (i) is that a phase transition can occur as $\beta$ is varied at constant $H$ only if $H$ is a limit point of zeros of $\Xi$ (which can happen only if $\operatorname{Re} H=0$ ); this supplements Lee and Yang's result that the same condition is necessary for a phase transition when $H$ is varied at constant $\beta$.

For a lattice or continuum gas with non-negative interaction potential (corresponding, in the lattice case, to an Ising antiferromagnet), similar results are shown to hold provided $\beta>0$ and the complex fugacity $z$ is less than the radius of convergence of the Mayer $z$ expansion; for the continuum gas, however, $n_{s}$ and $u_{s}$ must be replaced by their values integrated over small volumes surrounding each of the points $\boldsymbol{x}_{2}, \ldots, \boldsymbol{x}_{s}$.

It is shown that the pressure $p$ is analytic in both $\beta$ and $z$, if it is analytic in $z$ at fixed $\beta$ over a suitable range of values of $\beta$ and $z$, and further that, except for continuum systems without hard cores, $p, n_{s}$ and $u_{s}$ have convergent Maclaurin expansions in $\beta$ for small enough $z$.
\end{abstract}

\section{Introduction}

Various authors $[1,2,3]$ have studied the analytic properties of the thermodynamic pressure $p \equiv \beta^{-1} \lim _{V \rightarrow \infty} V^{-1} \log \Xi(\beta, z, V)$ and $s$-body distribution functions $n_{s}\left(\boldsymbol{x}_{1}, \ldots, \boldsymbol{x}_{s}\right)$ of a classical system of particles with two-body interaction potential $\varphi(r)$, in a volume $V$, as the fugacity $z$ varies at a fixed positive temperature $1 / k \beta$. They showed how to specify a disk

$$
|z|<D(\beta)
$$

* Supported by the U.S. Air Force Office of Scientific Research under grant no. AF 68-1416. 
in the complex $z$ plane, with radius depending on $\beta$, within which $p$ and $n_{s}\left(\boldsymbol{x}_{1}, \ldots, \boldsymbol{x}_{s}\right)$ are analytic functions of $z$. RUELLE [4] pointed out that these results also apply when $\beta$ is complex, provided its real part is positive, and therefore furnish information about the nature of the functional dependence on $\beta$ too: in particular, he showed that if $|z|<D(\beta)$ and $\beta>0$ then $p$ and $n_{s}\left(\boldsymbol{x}_{1}, \ldots, \boldsymbol{x}_{s}\right)$ are analytic functions of the two variables $\beta$ and $z$. In the same paper, RUELLE also proved the related result that if $|z|<D(\beta)$ then the Ursell functions, defined [5] by

$$
\begin{aligned}
u_{1}\left(\boldsymbol{x}_{1}\right)= & n_{1}\left(\boldsymbol{x}_{1}\right) \\
u_{2}\left(\boldsymbol{x}_{1}, \boldsymbol{x}_{2}\right)= & n_{2}\left(\boldsymbol{x}_{1}, \boldsymbol{x}_{2}\right)-n_{1}\left(\boldsymbol{x}_{1}\right) n_{1}\left(\boldsymbol{x}_{2}\right) \\
u_{3}\left(\boldsymbol{x}_{1}, \boldsymbol{x}_{2}, \boldsymbol{x}_{3}\right)= & n_{3}\left(\boldsymbol{x}_{1}, \boldsymbol{x}_{2}, \boldsymbol{x}_{3}\right)-n_{2}\left(\boldsymbol{x}_{1}, \boldsymbol{x}_{2}\right) n_{1}\left(\boldsymbol{x}_{3}\right)-n_{2}\left(\boldsymbol{x}_{1}, \boldsymbol{x}_{3}\right) n_{1}\left(\boldsymbol{x}_{2}\right) \\
& -n_{2}\left(\boldsymbol{x}_{2}, \boldsymbol{x}_{3}\right) n_{1}\left(\boldsymbol{x}_{1}\right)+2 n_{1}\left(\boldsymbol{x}_{1}\right) n_{1}\left(\boldsymbol{x}_{2}\right) n_{1}\left(\boldsymbol{x}_{3}\right), \text { etc. }
\end{aligned}
$$

have the cluster property

$$
\int \cdots \int\left|u_{s}\left(\boldsymbol{x}_{1}, \ldots, \boldsymbol{x}_{s}\right)\right| d^{v} \boldsymbol{x}_{2} \ldots d^{v} \boldsymbol{x}_{s}<\infty
$$

where $v$ is the number of space dimensions. In the present paper we consider some situations where similar analyticity and clustering properties can be proved for values of $z$ and $\beta$ that do not satisfy the conditions (1).

Our discussion of analyticity is mainly based (like RuELLE's) on the fugacity expansion

$$
\beta p(\beta, z)=z+-\sum_{l=2}^{\infty} b_{l}(\beta) z^{l}
$$

and the corresponding expansions for $n_{s}\left(\boldsymbol{x}_{1}, \ldots, \boldsymbol{x}_{s}\right)$. The coefficients in the series (4) are the Mayer cluster integrals, of which the simplest is

$$
b_{2}(\beta)=\frac{1}{2} \int\left[e^{-\beta \varphi(r)}-1\right] d^{\nu} \boldsymbol{r} .
$$

Since the integrand is analytic in $\beta$, the integral is also analytic in the half-plane $\operatorname{Re} \beta>0$ where the integral converges, provided $\varphi(r)$ satisfies suitable conditions. The same applies to the higher cluster integrals, so that every term in the series (4) is analytic in $\beta$ and $z$. One way of showing that $p(\beta, z)$ itself is analytic, say when $\beta=\beta_{0}$ and $z=z_{0}$ with $\beta_{0}>0$, is to show that this series of analytic functions converges uniformly in a neighborhood of the point $\left(\beta_{0}, z_{0}\right)$ in the four-dimensional space of the two complex variables $\beta$ and $z$. It is not sufficient, however, to show only that the series converges for real values of $\beta$ near $\beta=\beta_{0}$ and complex values of $z$ near $z=z_{0}$; for example, the series

$$
\sum_{l} z^{l} \cos \left(l^{2} \beta\right)
$$


converges if $\beta$ is real and $|z|<1$, but its sum is not analytic in $\beta$. (To see this, we may note that if the function defined by this series is expanded in powers of $\beta$, the coefficient of $\beta^{4 n}$ is (for $z>0$ )

$$
\sum_{l} z^{l} l^{8 n} /(4 n) !>z^{4 n}(4 n)^{8 n} /(4 n)^{4 n}=(4 n z)^{4 n},
$$

from which it follows that this expansion in powers of $\beta$ has vanishing radius of convergence.)

The first result of this paper, given in Section II, is to show that for systems with hard cores the thermodynamic functions and distribution functions do have convergent series expansions in powers of $\beta$ (that is, they are analytic in $\beta$ at $\beta=0$ ). To deal with values of $\beta$ that are not near $\beta=0$, we next prove (Section III) a lemma giving conditions under which analyticity in $z$ at fixed $\beta$ implies analyticity in both variables. In Section IV we apply this theorem to the pressure, and in Sections $\mathrm{V}$ and VI to the distribution functions (for which it is first necessary to prove analyticity in $z$ at fixed $\beta$; the difficulty of doing this restricts the class of systems to which our results about distribution functions apply). Finally in Sections VII and VIII we discuss the clustering tendency, one expression of which is (3), for the Ursell functions formed from the distribution functions considered in Sections V and VI.

We shall assume that the interaction between the particles is determined by a two-body potential $\varphi(r)$ satisfying the conditions [4]

(i) convergence: $\varphi(r)$ is bounded below, satisfies

$$
\left.\begin{array}{ll}
\int_{r>a}|\varphi(r)| d^{v} \boldsymbol{r}<\infty & \text { (continuum) } \\
\sum_{r>a}|\varphi(r)|<\infty & \text { (lattice) }
\end{array}\right\}
$$

for some positive $a$, and is bounded above for $r>a$; and

(ii) stability:

$$
\Phi=-\inf _{n} \inf _{\boldsymbol{x}_{1}, \ldots, \boldsymbol{x}_{n}} \frac{1}{n} \sum_{1 \leqq i<j \leqq n} \varphi\left(\left|\boldsymbol{x}_{i}-\boldsymbol{x}_{j}\right|\right)<\infty .
$$

The convergence condition ensures that cluster integrals such as (5) converge for all values of $\beta$ in the half plane $\operatorname{Re} \beta>0$. It also ensures that they are analytic functions of $\beta$ in this half plane; to indicate how this is proved we show that the integral (5) is analytic in $\beta$ by showing [6] that its first derivative,

$$
d b_{2} / d \beta \equiv \lim _{|h| \rightarrow 0}\left[b_{2}(\beta+h)-b_{2}(\beta)\right] / h
$$

where $h_{l}=h \mid e^{i l}$ and $\operatorname{Re} \beta>0$, is independent of $\theta$. Adding

$$
\frac{1}{2} \int e^{-\beta \varphi(r)} \varphi(r) d^{v} v
$$


to both sides and using the definition (5) we obtain $d b_{2} / d \beta+\frac{1}{2} \int e^{-\beta \varphi(r)} \varphi(r) d^{v} \boldsymbol{v}=\frac{1}{2} \lim \int d^{v} \boldsymbol{r} e^{-\beta \varphi(r)} \int_{0}^{\varphi(r)} d t\left[1-e^{-h t}\right]$.

Since

$$
\left|1-e^{-h t}\right|=\left|h t \int_{0}^{1} e^{-s h t} d s\right| \leqq|h t| e^{|h t|},
$$

the absolute value of the right side of the last equation is at most

$$
\frac{1}{2} \lim |h| \int d^{v} \boldsymbol{r} e^{-\beta \varphi(r)} \varphi(r)^{2} e^{|h \varphi(r)|} .
$$

By (6), the integral converges if $\operatorname{Re} \beta>|h|$, and is bounded in some neighbourhood of $h=0$ provided $\operatorname{Re} \beta>0$; therefore the limit is zero for any $\theta$, showing that $b_{2}(\beta)$ is indeed analytic in the half plane $\operatorname{Re} \beta>0$.

The stability condition can be used $[3,4]$ to obtain bounds on the cluster integrals in the half plane $\operatorname{Re} \beta>0$; these bounds are

where

$$
\left|b_{l}(\beta)\right| \leqq e^{2(l-2) \Phi R e \beta}[l B(\beta)]^{l-1} / l ! l
$$

$$
B(\beta) \equiv \int\left|e^{-\beta \varphi(r)}-1\right| d^{v} \boldsymbol{r}, \quad(\operatorname{Re} \beta>0) .
$$

For a lattice system, the integral becomes a sum. The uniform convergence of the pressure series (4) can therefore be proved for any compact set in the region $E$ of $(\beta, z)$ space defined by the inequalities

$$
\begin{gathered}
\operatorname{Re} \beta>0 \\
|z|<D(\beta) \equiv 1 /\left[e B(\beta) e^{2 \Phi R e \beta}\right]
\end{gathered}
$$

and consequently, since every term in the series is analytic, the sum $p(\beta, z)$ is also analytic in both variables within $E$. An analogous argument applied to the fugacity expansion for the distribution functions shows that $n_{s}\left(\boldsymbol{x}_{1}, \ldots, \boldsymbol{x}_{s}\right)$ too is analytic when $(\beta, z)$ is in $E$ for any $s$ and any $\boldsymbol{x}_{1}, \boldsymbol{x}_{2}, \ldots, \boldsymbol{x}_{s}$.

For potentials without hard cores, the region $E$ includes some points for all values of $|z|$; but with hard cores, there is an upper bound on the possible values of $|z|$ (see Eq. (20) below).

\section{Power Series Expansion in $\boldsymbol{\beta}$}

Since $\beta=0$ lies on the boundary of $E$, there is no general reason to expect a series expansion of $p$ or $n_{s}$ in powers of $\beta$ to converge. For hardcore potentials, however, we can show that such expansions do converge for suitable values of $z$. The method is to show by analytic continuation that $p$ and $n_{s}$ are also regular in a region $E^{\prime}$ similar to $E$ but with $\operatorname{Re} \beta \leqq 0$. 
A hard-core potential is defined by the condition

$$
\left.\begin{array}{lll}
\varphi(r)=+\infty & \text { for } & r \leqq a \\
\text { and }<\infty & \text { for } & r>a
\end{array}\right\}
$$

where $\mathrm{a}$ is a positive constant, the diameter of the hard core. For such potentials, it is convenient to replace (6) by the slightly stronger condition

$$
|\varphi(r)|<\psi(r) \text { for } r>a
$$

where $\psi(r)$ is a non-increasing function satisfying

$$
\int_{r>a} \psi(r) d^{v} \boldsymbol{r}<\infty
$$

(or the corresponding condition for a lattice). The conditions (11) and (12) together ensure that the stability condition (7) is satisfied [7], both by $\varphi(r)$ itself and by the potential $\varphi^{\prime}(r)$ defined by

$$
\varphi^{\prime}(r) \equiv\left\{\begin{array}{lll}
+\infty & \text { if } & r \leqq a \\
-\varphi(r) & \text { if } & r>a
\end{array}\right\}
$$

It was pointed out by Jones [8] that for hard-core potentials the analytic continuation of the grand partition function $\Xi(\beta, z, V)$ into the left half of the $\beta$ plane is given by

$$
\Xi(\beta, z, V)=\Xi^{\prime}(-\beta, z, V)
$$

where $\Xi$ refers to the potential $\varphi$ and $\Xi^{\prime}$ to $\varphi^{\prime}$. A similar method can be used to continue the functions $p$ and $n_{s}$ into the left half-plane. For brevity we consider only the function $p$, but the method and results for $n_{s}$ are just the same.

Writing the integral (5) in the form

$$
b_{2}(\beta)=-\frac{1}{2} K_{v}(a)+\frac{1}{2} \int_{r>a}\left[e^{-\beta \varphi(r)}-1\right] d^{v} v^{v}
$$

where $K_{v}(a)$ is the volume of a $\nu$-dimensional sphere of radius $a$, and using (12), we see that $b_{2}(\beta)$ is an entire function of $\beta$ and satisfies

$$
b_{2}(\beta)=b_{2}^{\prime}(-\beta)
$$

where $b_{2}^{\prime}$ refers to the potential $\varphi^{\prime}$. The same argument applies to the higher cluster integrals, and it follows that the function of $\beta$ and $z$ defined by the series (4) is regular throughout any region in $(\beta, z)$-space having the property that the series converges uniformly on all its compact subsets. The region $E$ defined in (10) has this property, and so does the region $E \cup E^{\prime}$ with $E^{\prime}$ defined by

$$
\begin{aligned}
\operatorname{Re} \beta & \leqq 0 \\
|z| & <1 /\left\{e B^{\prime}(-\beta) \exp \left[-2 \Phi^{\prime} \operatorname{Re} \beta\right]\right\}
\end{aligned}
$$


where $\Phi^{\prime}$ is defined by (7) with $\varphi^{\prime}$ replacing $\varphi$, and

$$
B^{\prime}(-\beta) \equiv K_{v}(a)+\int_{r>a}\left|e^{-\beta \varphi(r)}-1\right| d^{v} \boldsymbol{r} .
$$

The fact that the conditions (18) are sufficient to ensure uniform convergence of the series (4) on any compact subset of $E^{\prime}$ can be verified using the fact that $b_{l}(\beta)=b_{l}^{\prime}(-\beta)$, together with the analogue of (8) for the potential $\varphi^{\prime}$.

Suppose now that $z$ is given a fixed value, say $z_{0}$, satisfying

$$
\left|z_{0}\right|<1 / e B^{\prime}(0)=1 / e K_{\nu}(a) ;
$$

then the set of values of $\beta$ for which $\left(\beta, z_{0}\right) \in E \cup E^{\prime}$ includes a neighborhood of the origin in the $\beta$-plane, and so $p\left(\beta, z_{0}\right)$ can be expanded as a power series in $\beta$ with a positive radius of convergence (which will depend on $z_{0}$ ). Such power series in $\beta$ are used in various branches of statistical mechanics [9], but had not previously been proved to be convergent for any fluid system. For lattice systems, a proof implying such convergence for sufficiently small $|\beta|$ and small $|z|$ has been given by Gallavotti and Mrracle-Sote [24], and analyticity for small $|\beta|$ and any $z>0$ is proved in [12].

\section{Extension of the Domain of Analyticity}

In the rest of this paper we shall be interested in showing that $p$ and $n_{s}$ are analytic functions of $\beta$ and $z$ for points outside the region $E$ (or in the case of hard cores $\left.E \cup E^{\prime}\right)$, particularly for those points $(\beta, z)$, with $\beta>0$, where it is known from other considerations (such as the Lee-Yang theorem [10]) that the series (4) converges. The following lemma makes it possible to prove analyticity in both $\beta$ and $z$ at such points:

Lemma. Let $C$ be a closed disk in the $z$-plane and $K$ the closure of a bounded simply connected region having $C$ in its interior (see Fig. 1). Let $I$ be a closed interval of the real $\beta$ axis and $S$ a closed semicircle in the upper half of the $\beta$-plane, whose diameter is $I$. Let $F^{\top}(\beta, z)$ be a function with the following properties:

(i) $F(\beta, z)$ is analytic in both variables for all $\beta$ in $S$ and $z$ in $C$ - that is, it is analytic in some neighborhood of the closed set $S \times C$ in $(\beta, z)$ space; (21)

(ii) $F(\beta, z)$ is analytic in $z$ (at fixed $\beta$ ) for all $\beta$ in $I$ and $z$ in $K$ - that $i$ s, for each $\beta$ in $I$ it is analytic in some neighborhood of the closed set $K$ in the z-plane;

(iii) there is a constant $M^{\prime}$ such that

$$
|F(\beta, z)|<M^{\prime}
$$

for all $\beta$ in $I$ and $z$ in $K$. Then $F(\beta, z)$ is analytic in both variables for all $\beta$ in $I$ and all $z$ in $K^{\prime}$, the interior of $K$ - that is, it is analytic in some neighborhood of the set $I \times K^{\prime}$ in $(\beta, z)$ space. 
Proof. By Riemann's mapping theorem [11], there is a conformal transformation

$$
t=f(z)
$$

that maps the interior of $K$ in the $z$ plane on to the open unit disk in the $t$-plane and the point $z=0$ into the point $t=0$. Our hypothesis (21) then implies that $F(\beta, z)$ has a series expansion in powers of $t$ :

$$
F(\beta, z)=\sum_{n} G_{n}(\beta) f(z)^{n}=\sum_{n} G_{n}(\beta) t^{n}
$$
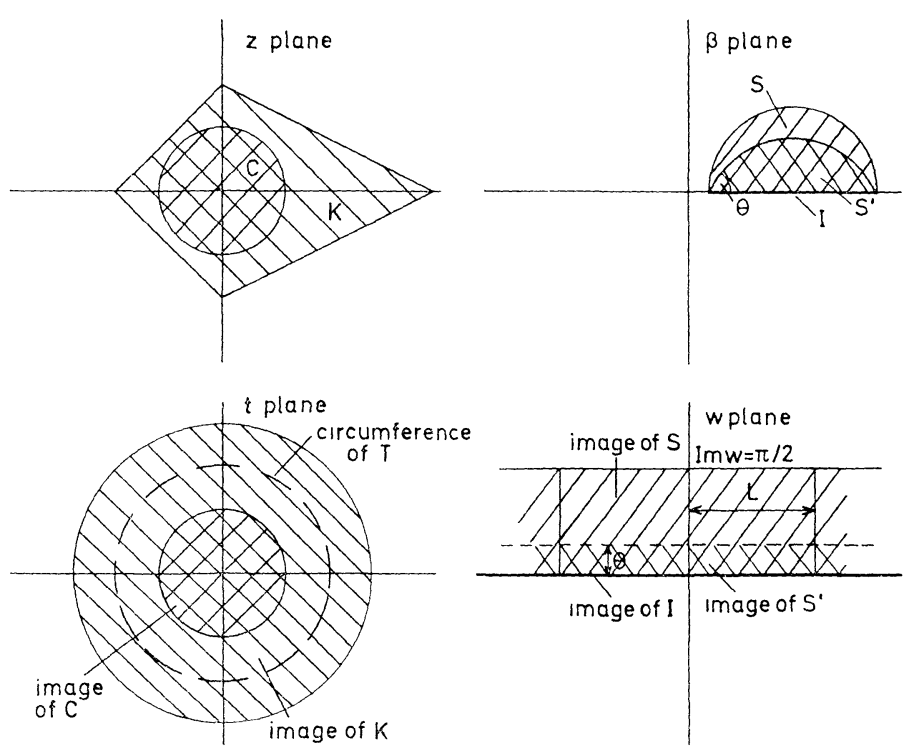

Fig. 1

whose coefficients are analytic functions of $\beta$ throughout $S$. If $M^{\prime \prime}$ is the largest value taken by $|F(\beta, z)|$ for $\beta$ in $S$ and $z$ in $C$, then Cauchy's inequality gives

$$
\left|G_{n}(\beta)\right| \leqq M^{\prime \prime} / r^{n} \quad \text { if } \quad \beta \in S
$$

where $r$ is the least distance from $t=0$ to the image of the boundary of C. Moreover, by (22) and (23), Cauchy's inequality applied to the unit circle in the $t$-plane gives

$$
\left|G_{n}(\beta)\right| \leqq M^{\prime} \quad \text { if } \quad \beta \in I .
$$

Without loss of generality we may take the end points of $I$ to be -1 and +1 ; then the conformal transformation

$$
w=\log [(1+\beta) /(1-\beta)] \quad \text { or } \quad \beta=\tanh \frac{1}{2} w
$$


maps $I$ on to the line $\operatorname{Im} w=0$ and $S$ on to the infinite strip

$$
0 \leqq \operatorname{Im} w \leqq \frac{1}{2} \pi
$$

We shall apply the maximum modulus principle to the function

$$
H_{n}(w) \equiv G_{n}\left(\tanh \frac{1}{2} w\right) \exp \left(-2 i w n \pi^{-1} \log r\right)
$$

in a rectangular region bounded by the $\operatorname{lines} \operatorname{Im} w=0, \operatorname{Im} w=\frac{1}{2} \pi$, $\operatorname{Re} w= \pm L$, where $L$ is arbitrarily large. From (25) we see that $\left|H_{n}(w)\right|$ is bounded within the rectangle and satisfies

$$
\left|H_{n}(w)\right| \leqq M \equiv \max \left(M^{\prime}, M^{\prime \prime}\right)
$$

on the sides $\operatorname{Im} w=0$ and $\operatorname{Im} w=\frac{1}{2} \pi$. On the right-hand end of the rectangle we have, by (27), $|1-\beta|=|1+\beta| e^{-L}$. Since $|1+\beta| \leqq 2+|1-\beta|$ this implies that, on the right-hand end,

$$
|1-\beta| \leqq 2 /\left(e^{L}-1\right) \text {. }
$$

It follows by (28) that the maximum value of $\left|H_{n}(w)\right|$ on the right-hand end of the rectangle cannot exceed the maximum value of $\left|G_{n}(\beta)\right|$ in the $\beta$-plane disk defined by (30). In the limit $L \rightarrow \infty$, this disk shrinks to a point, and the limiting maximum value of $\left|G_{n}(\beta)\right|$ in it is at most $M^{\prime}$ by (26), since $G_{n}(\beta)$ is analytic and therefore continuous at $\beta=1$. A similar argument applies to the other end of the rectangle which corresponds to $\beta=-1$, so that as $L \rightarrow \infty$ the maximum value of $\left\{H_{n}(w)\right\}$ on the ends of the rectangle tends to a limit not exceeding $M^{\prime}$. Combining this with (29) and using the maximum modulus principle we obtain

$$
\left|H_{n}(w)\right| \leqq M \quad \text { if } \quad 0 \leqq \operatorname{Im} w \leqq \frac{1}{2} \pi .
$$

By (28), this is equivalent to

$$
\left|G_{n}\left(\tanh \frac{1}{2} w\right)\right| \leqq M / r^{2 n \operatorname{Im} w / \pi}
$$

Let $r^{\prime}$ be any number satisfying $0<r^{\prime}<1$, and let $T$ be the closed disk $|t| \leqq r^{\prime}$ in the $t$-plane. When $t \in T$, (32) shows that the series (24) for $F(\beta, z)$ is majorized by the geometric series

$$
M \sum_{n}\left[r^{\prime} \mid r^{2 \operatorname{Im} w / \pi}\right]^{n}
$$

Since $r^{\prime}<1$ and $r<1$ we can find a positive number $\theta$ small enough to make

$$
r^{\prime}<r^{2 \theta / \pi} \text { and } 0<\theta<\frac{1}{2} \pi
$$

and then it follows by (27) that the series (33) is uniformly convergent if $\beta$ satisfies

$$
0 \leqq \operatorname{Im} \log [(1+\beta) /(1-\beta)] \leqq \theta .
$$


The closed region of the $\beta$-plane defined by $(35)$ is a segment of a circle with base $I$ and angle $\theta$; it is a subregion of $S$ and we shall call it $S^{\prime}$. The majorization (33) implies that the series (24) converges uniformly for $t \in T$ and $\beta \in S^{\prime}$, and since the terms of this series are analytic the sum, $F(\beta, z)$, is also analytic. Given any $z$ inside $K$ we can always choose $r^{\prime}$ so that the disk $T$ contains the image of $z$; then we choose $\theta$ to satisfy (34), and since the closed region $S$ defined by (35) includes $I$, the analyticity of $F(\beta, z)$ for all $\beta$ in $I$ and $z$ inside $K$ is proved, Q.E.D.

Since this is a purely mathematical result there is no need to interpret $\beta$ as recriprocal temperature or $z$ as fugacity. This freedom is useful in some applications of the theorem; for example in Section IX we replace $\beta$ by a variable $\lambda$ describing the strength of a non-uniform external potential.

\section{Analyticity of the Pressure}

In this section we use the lemma of Section III to show that $p(\beta, z)$ is analytic in both variables at the point $\left(\beta_{0}, z_{0}\right)$ provided that the following condition is satisfied:

There exist a closed interval $I$ of the real $\beta$ axis, containing $\beta=\beta_{0}$, and a bounded simply-connected region $J$ in the $z$-plane, containing both $z=z_{0}$ and $z=0$, such that any closed sub-region of $J$ is free of zeros of $\Xi(\beta, z, V)$ for all $\beta$ in $I$ and all sufficiently large $V$.

An important case where this condition is satisfied is the Ising spin system with purely ferromagnetic interactions. Here, instead of $z$, we use the variable

$$
\zeta=e^{-2 m H \beta}
$$

where $H$ is the applied magnetic field and $m$ is the magnetic moment of each spin. LEE and $\mathrm{YANG}_{\mathrm{AN}}[10]^{1}$ have shown that, for $\beta>0$, all the zeros of the Ising spin partition function $\Xi_{s}(\beta, \zeta, V)$, which is proportional to the corresponding lattice gas grand partition function $\Xi$, lie on the unit circle in the $\zeta$ plane, and hence we may always take $J$ to be the open unit disk and $I$ any segment of the positive real axis; by the symmetry of the Ising model under the field reversal transformation $\zeta \rightarrow 1 / \zeta$, our results also apply to the region $|\zeta|>1$.

For a suitable choice of $I$, it may also happen that some points on the unit circle are not limit points of zeros of $\Xi(\beta, \zeta, V)$ as $V \rightarrow \infty$ with $\beta \in I$ : if so, these non-limit points form a set of open arcs which we call windows. In particular, since the pressure and distribution functions are known [12] to be analytic at $\zeta=1$ for small $\beta>0$, we expect the point $\zeta=1$ to belong to such a window if $I$ includes only small values of $\beta$. If windows do exist then $J$ may be extended beyond the unit circle into the region $|\zeta|>1$ through any one of the windows.

1 These authors use the symbols $y, z$ where we use $z, \zeta$.

8 Commun. math. Phys., Vol.11 
Apart from surface effects the Ising ferromagnet is [10] isomorphic to a lattice gas with an attractive interaction potential (that is, with $\varphi(r) \leqq 0$ for $r \neq 0$, but $\varphi(0)=+\infty)$ and fugacity

where

$$
z=\zeta e^{-\alpha \beta}
$$

$$
\alpha \equiv \lim _{V \rightarrow \infty} \alpha(\boldsymbol{r}, V) \equiv \lim _{V \rightarrow \infty}\left[-\frac{1}{2} \sum_{\boldsymbol{r}^{\prime} \in V, \neq \boldsymbol{r}} \varphi\left(\boldsymbol{r}^{\prime}-\boldsymbol{r}\right)\right]=-\frac{1}{2} \sum_{\boldsymbol{r}} \varphi(\boldsymbol{r}),
$$

the last sum covering all points on an infinite lattice except $\boldsymbol{r}=0$; all our results for the Ising ferromagnet can therefore be re-interpreted in terms of such a lattice gas.

To prove that $p(\beta, z)$ is analytic in both variables for all cases where (36) is satisfied, we apply the lemma of Section III to the function $F(\beta, z)=\exp [\beta p(\beta, z)]$. (For the Ising ferromagnet, read $\zeta$ in place of $z$ throughout, unless a separate mention of $\zeta$ is made.) The regions $I, C$, etc., in the $\beta$ and $z$ planes must be chosen in accordance with conditions (21), (22), and (23). We choose $I$ to be the interval referred to in (36); let it be $\beta^{\prime} \leqq \beta \leqq \beta^{\prime \prime}$. We choose $C$ to be any disk $|z| \leqq r(|\zeta| \leqq r$ for the ferromagnet) whose radius satisfies

$$
0<r<1 /[e B(\beta) \exp (2 \Phi \operatorname{Re} \beta)]
$$

for all $\beta$ in $S$. Such a choice is always possible, since the function $B(\beta)$ is bounded in $S$. Then, it follows from (39) and (10) that the region $S \times C$ in $(\beta, z)$ space or $(\beta, \zeta)$ space lies inside the region $E$, so that condition (21) of the lemma is satisfied. (It might appear from (38) that the larger region $|\zeta|<r e^{\alpha \beta}$ can be used for the Ising ferromagnet in place of $C$, but because the correspondence between Ising model and lattice gas is not perfect at the boundaries the proof does not go through so easily for the larger region.) To satisfy the second condition of the lemma, we choose $K$ to be the closure of a simply-connected region, lying inside $J$ and containing the points $z=0$ and $z=z_{0}$. By YANG and LEE's theorem [13], the function $\beta p(\beta, z)$ is analytic in $z$ throughout $J$ for all $\beta \in I$, so that the condition (22) is satisfied. To show that the third condition, Eq. (23), is satisfied, we start from the thermodynamic interpretation of the grand partition function in the form

$$
\exp [\beta p(\beta, z)]=\lim _{V \rightarrow \infty}[\Xi(\beta, z, V)]^{1 / V} .
$$

Applying the definitions of $F(\beta, z)$ and of $\Xi(\beta, z, V)$, and taking absolute values with the help of (7), we find that

$$
|F(\beta, z)| \leqq \lim _{V \rightarrow \infty}\left[\left[1+\sum_{N}\left|z e^{\beta \Phi}\right|^{N} V^{N} \mid N !\right]^{1 / V}=\exp \left(\left|z e^{\beta \Phi}\right|\right),\right.
$$

so that $|F(\beta, z)|$ is bounded on the bounded set $I \times J$. 
Since all three conditions of the lemma are satisfied, we conclude that $F(\beta, z)=\exp [\beta p(\beta, z)]$ (or $\exp [\beta p(\beta, \zeta)]$ for the Ising ferromagnet) is indeed analytic in both variables when the condition (36) is satisfied. In the case of an Ising ferromagnet, a sufficient condition is simply that the magnetic field should not vanish. Since $F(\beta, z)$ cannot vanish in $I \times J$, the analyticity of $p(\beta, z)$ in $I \times J$ follows.

This result has the corollary that the thermodynamic energy and specific heat per unit volume, $-\partial(\beta p) / \partial \beta$ and $k \beta^{2} \partial(\beta p) / \partial \beta^{2}$, are also analytic in $\beta$ and $z$ under the same conditions.

\section{Analytic Properties of Distribution Functions: Ising Ferromagnet}

For a system of particles in a finite volume $V$, the $s$-particle distribution function may be defined as

$n_{s}\left(\boldsymbol{X}_{s} ; \beta, z ; V\right)=\sum_{N=s}^{\infty} \frac{z^{N}}{(N-s) !} \int_{V} \cdots \int_{V} e^{-\beta U_{N}} d \boldsymbol{x}_{s+1} \ldots d \boldsymbol{x}_{N} / \Xi(\beta, z ; V)$

where $\boldsymbol{X}_{s} \equiv\left(\boldsymbol{x}_{1}, \ldots, \boldsymbol{x}_{s}\right)$ and $U_{N}^{-}$is the potential energy of the $N$-particle configuration $x_{1}, \ldots, x_{N}$. The corresponding infinite-volume distribution function is defined by

$$
n_{s}\left(\boldsymbol{X}_{s} ; \beta, z\right) \equiv \lim _{V \rightarrow \infty} n_{s}\left(\boldsymbol{X}_{s} ; \beta, z ; V\right)
$$

if the limit exists. It is usual to take this limit by making the entire boundary of $V$ recede to infinity in all directions, but our results also apply to the case where only a part of it recedes to infinity. It has been shown elsewhere $[2,4]$ that the limit does exist, and defines an analytic function of $\beta$ and $z$, inside the region $E$ of $(\beta, z)$ space. To extend these results to a larger region of $(\beta, z)$ space, we shall make use of Vitali's theorem [14]. This theorem asserts that if a sequence of analytic functions of one variable is bounded on a compact set, which in our applications will normally be $K$, and tends to a limit at an infinite number of points of this set, then the sequence tends to a limit at all points of the set and the limiting function is analytic. In our case, since both numerator and denominator of (41) are entire functions of $z$, the quotient is analytic in any region of the $z$-plane that is free of zeros of $\Xi$; in particular, given any compact subset of the region $J$ described in (36), this quotient is analytic on the subset for all sufficiently large $V$ and all $\beta$ in $I$. Moreover $[3,4]$ the sequence $(42)$ converges, for fixed $\beta$, throughout the disk $|z|<D(\beta)$ and therefore, for all $\beta$ in $I$, throughout the disk $C$ defined in (39). To justify the use of Vitali's theorem it thus remains to show that $n_{s}\left(\boldsymbol{X}_{s} ; \beta, z ; V\right)$ is bounded for fixed $\beta \in I$ for all sufficiently large $V$ and all $z$ in a compact set, say $K$, that is contained in $J$ and shares an 
infinite number of points with $C$. We have not succeeded in proving a useful boundedness property of $n_{s}$ for the general class of potentials defined by (6) and (7) but we give proofs here for two special classes.

The first special type of system we consider is the Ising ferromagnet, in which (for $\zeta>0, \beta>0) n_{s}\left(\boldsymbol{X}_{s} ; \beta, \zeta\right)$ is the probability of finding $s$ down-spins at the sites $\boldsymbol{x}_{1}, \ldots, \boldsymbol{x}_{s}$. We consider first the function $n_{1}\left(\boldsymbol{x}_{1}\right)$. Following LEE and YANG [10] we consider a generalized grand partition function for the equivalent lattice gas, with a different fugacity $z_{i}$ (or magnetic field $H_{i}$ ) acting at each of the $V$ lattice sites, which we label $1,2, \ldots, V$ with the site $x_{1}$ labelled 1 . This generalized grand partition function is a multinomial of degree 1 in each of the $V$ variables $\zeta_{1}, \ldots, \zeta_{V}$ where $\zeta_{i} \equiv \exp \left(-2 m H_{i} \beta\right) \equiv z_{i} \exp \left(\alpha_{i} \beta\right)$ and $\alpha_{i} \equiv \alpha\left(\boldsymbol{x}_{i}, V\right)$ as defined just after (38); it may therefore be written in the form

$$
\Xi\left(\beta, \zeta_{1}, \ldots, \zeta_{V} ; V\right)=P+\zeta_{1} Q
$$

where $P$ and $Q$ depend on $\beta, \zeta_{2}, \ldots, \zeta_{V}, V$ only. Setting $\zeta_{2}=\zeta_{3}=\cdots$ $=\zeta_{V} \equiv \zeta$ and denoting the resulting values of $P$ and $Q$ by $P(\zeta, V)$ and $Q(\zeta, V)$ we obtain

$$
\Xi\left(\beta, \zeta_{1}, \zeta, \ldots, \zeta ; V\right)=P(\zeta, V)+\zeta_{1} Q(\zeta, V)
$$

In this notation the value of $n_{1}$ at the lattice site labelled 1 is

where

$$
n_{1}(1 ; \beta, \zeta ; V)=f(\zeta, V) /[1+f(\zeta, V)]
$$

$$
f(\zeta, V)=\zeta Q(\zeta, V) / P(\zeta, V) .
$$

Since $\Xi(\beta, \zeta ; V)$ is a polynomial in $\zeta$ of degree $V$, it follows from (44) that $Q$ is a polynomial of degree $V-1$; it may therefore be factorized in the form

$$
Q(\zeta, V)=\text { const. } \prod_{i=1}^{V-1}\left(\zeta-q_{i}(V)\right)
$$

where $q_{1}(V), \ldots, q_{V-1}(V)$ are its zeros. By virtue of the symmetry of the Ising model under magnetic field reversal, the polynomials $P$ and $Q$ are related by

$$
P(\zeta, V)=\zeta^{V-1} Q\left(\zeta^{-1}, V\right) .
$$

Substituting (47) and (48) into (46) we obtain the factorization

$$
f(\zeta, V)=\zeta \prod_{i=1}^{V-1} \frac{\zeta-q_{i}(V)}{1-\zeta q_{i}(V)}=\zeta \prod_{i=1}^{V-1} \frac{\zeta-q_{i}(V)}{1-\zeta q_{i}^{*}(V)} .
$$

The last step is justified by the fact that $Q(\zeta, V)$ has real coefficients, so that its complex roots occur in conjugate pairs.

For ferromagnetic interactions, we can show that all the zeros of $f(\zeta, V)$, and none of the poles, are inside the unit circle. To do this we use a lemma proved by LEE and YANG [10], which shows that 
$\Xi\left(\beta, \zeta_{1}, \ldots, \zeta_{V} ; V\right)$ cannot vanish if $\left|\zeta_{i}\right|>1$ for all $i$. It follows from this that if $\left|\zeta_{1}\right|>1$ and $|\zeta|>1$ then [by (44) and (46)] $f(\zeta, V) / \zeta$ cannot take the value $-1 / \zeta_{1}$, so that if $|\zeta|>1$ then $|f(\zeta, V) / \zeta|$ must either be at least 1 or else 0 . Since the value 0 is impossible by continuity we conclude that

$$
|f(\zeta, V)| \geqq|\zeta| \text { if }|\zeta|>1
$$

This implies, by (49), that

so that

$$
\left|q_{i}(V)\right| \leqq 1 \quad i=1, \ldots, V-1
$$

and

$$
|f(\zeta, V)| \leqq|\zeta| \text { if }|\zeta|<1
$$

$$
|f(\zeta, V)|=1 \text { if }|\zeta|=1 .
$$

According to (45) we have

$$
f(\zeta, V)=n_{1}(1 ; \beta, \zeta ; V) /\left[1-n_{1}(1 ; \beta, \zeta ; V)\right] .
$$

The right side converges to a limit as $V \rightarrow \infty$ when $|\zeta|<r$ with $r$ satisfying (39), and so it follows by Vitali's theorem applied to $f(\zeta, V)$ that the function

$$
f(\zeta) \equiv \lim _{V \rightarrow \infty} f(\zeta, V)
$$

exists for $|\zeta|<1$ and is analytic. By virtue of the symmetry relation $f(1 / \zeta)=1 / f(\zeta, V)$, which is an immediate consequence of (49), we see that $f(\zeta)$ also exists for $|\zeta|>1$ and is meromorphic. It follows by (45) that $n_{1}(1 ; \beta, \zeta)$, defined in $(42)$, exists if $|\zeta| \neq=1$, and, incidentally, that it also satisfies the inequality ${ }^{2}$

$$
\operatorname{Re} n_{1}(1 ; \beta, \zeta) \lessgtr \frac{1}{2} \text { if }|\zeta| \lessgtr 1
$$

If there are windows on the unit circle, that is, sets of points that are not limit points of zeros of $\Xi(\beta, \zeta ; V)$ as $V \rightarrow \infty$, then we can also show that $n_{1}(1 ; \beta, \zeta)$ exists for these points. Being the complement of a set of limit points, the window itself is an open set. Let the arc $A_{1} A_{2}$, comprising the points $\zeta=e^{i \theta}$ with $\theta_{1} \leqq \theta \leqq \theta_{2}=\theta_{1}$, be a closed subset of the window. By suitable choice of $\theta_{1}$ and $\theta_{2}$ we may include any desired point of the window in $A_{1} A_{2}$.

For any $\zeta$ lying on the arc, every factor in the product (49) has modulus 1 and an argument that increases monotonically with $\theta$; it follows that $f$ itself has unit modulus and a monotonically increasing argument. For sufficiently large $V, \Xi(\beta, \zeta ; V)$ has no zeros on $A_{1} A_{2}$; this implies, by (44), that $f(\zeta, V)$ cannot take the value -1 on this are, and hence that arg $f$ cannot increase by as much as $2 \pi$ when $\zeta$ traverses the

2 The corresponding result for the thermodynamic density was found by BAKER [15]. 
arc. Since $\arg \zeta$ itself increases by $\theta_{2}-\theta_{1}$, as $\zeta$ traverses the arc, none of the quantities arg $\left[\left(\zeta-q_{i}\right) /\left(\mathbf{l}-\zeta q_{i}^{*}\right)\right]$ can increase by more than $2 \pi-\left(\theta_{2}-\theta_{1}\right)$. This condition is equivalent to the statement that none of the points $q_{i}$ can lie in the open circular segment $G^{\prime}$ bounded by the arc $A_{1} A_{2}$ and its chord (see Fig. 2); for if $q \in G^{\prime}$ then we have

$$
\begin{aligned}
& \arg \left(\frac{e^{i \theta_{2}}-q}{1-e^{i \theta_{2}} q^{*}} \cdot \frac{1-e^{i \theta_{1}} q^{*}}{e^{i \theta_{1}}-q}\right) \\
& \quad=\theta_{1}-\theta_{2}+2 \arg \left(\frac{e^{i \theta_{2}}-q}{e^{i \theta_{1}}-q}\right)>2 \pi-\left(\theta_{2}-\theta_{1}\right)
\end{aligned}
$$

so that the total contribution from the zero at $q$ to $\arg f$ exceeds the allowed maximum.

If $q$ lies in $G^{\prime}$ then $1 / q^{*}$ must lie in the crescent-shaped region, denoted by $G^{\prime \prime}$ in Fig. 2, obtained by inversion of $G^{\prime}$ in the unit circle. Since



Fig. 2

$f(\zeta, V)$ has no zeros in $G^{\prime}$ for large $V$, it also has no poles in $G^{\prime \prime}$; it is therefore regular in the region $G$ defined to consist of the union of the two regions $G^{\prime}$ and $G^{\prime \prime}$ and their common boundary, $A_{1} A_{2}$.

To apply Vitali's theorem to the sequence of analytic functions $f(\zeta, V)$ in $G$, we must show also that the sequence converges to an analytic function at an infinite number of points of $G$ and that it is uniformly bounded on any compact subset of $G$. That the first of these conditions is satisfied follows at once from the result of (55), which shows that the limit of the sequence is analytic at all points of $G^{\prime}$. To show that the condition of uniform boundedness is also satisfied, let $H$ be any compact subset of $G$, and $H^{\prime}$ its inversion consisting of all the points of the form $1 / \zeta^{*}$ with $\zeta \in H$. Since $G$ is its own inversion, $H^{\prime}$ too is a compact subset of the open set $G$ and so the distance from $H^{\prime}$ to the complement of $G$ is positive. Calling this distance $\delta$ we have, for any $i$,

$$
\left|1 / \zeta^{*}-q_{i}(V)\right| \geqq \delta>0 \quad \text { if } \quad \zeta \in H
$$


since $q_{i}(V)$ must be in the complement of $G$. Using $q_{i}$ as an abbreviation for $q_{i}(V)$ in (49), we obtain

$$
\begin{aligned}
|f(\zeta, V) / \zeta| & =\prod_{i}\left|\frac{\zeta-q_{i}}{1-\zeta q_{i}^{*}}\right|=\prod_{i}\left|\frac{1-q_{i}^{*} \mid \zeta^{*}}{1 / \zeta^{*}-q_{i}}\right| \\
& \leqq \prod_{i} \frac{\left|1-q_{i}^{*} q_{i}\right|+\left|q_{i}^{*}\right|\left|q_{i}-1 / \zeta^{*}\right|}{\left|q_{i}-1 / \zeta^{*}\right|}=\prod_{i}\left\{\left|q_{i}^{*}\right|+\frac{1-q_{i} q_{i}^{*}}{\left|q_{i}-1 / \zeta^{*}\right|}\right\} \\
& \leqq \prod_{i}\left\{1+\frac{1-q_{i} q_{i}^{*}}{\delta}\right\} \leqq \prod_{i}\left(q_{i} q_{i}^{*}\right)^{-1 / \delta}
\end{aligned}
$$

since $1+x / \delta<(1-x)^{-1 / \delta}$ for $0<x<1$, by the binomial theorem. From (49) and (46) we see that

$$
\prod_{i}\left|q_{i}\right|=Q(0, V) / P(0, V)
$$

which, by (43), is the coefficient of $\zeta_{1}$ in $\Xi\left(\beta, \zeta_{1}, \ldots, \zeta_{V} ; V\right)$. By virtue of the convergence condition (6) this coefficient $\left(x_{12} x_{13} \ldots x_{1 V}\right.$ in LEE and YANG's notation [10]) tends to a non-vanishing limit as $V \rightarrow \infty$; it follows that the last member of (59) is bounded for large $V$, and consequently $|f(\zeta, V)|$ is uniformly bounded for large $V$ and $\zeta \in H$.

Applying Vitali's theorem, we conclude that the function defined in (55) exists and is analytic not only for $|\zeta|<1$ but also throughout $G$, and in particular at all interior points of the are $A_{1} A_{2}$.

Applying Hurwitz's theorem [16] to the sequence $1+f(\zeta, V)$ we see also that $f(\zeta)$ cannot take the value -1 in $G$. It follows, by (45), that $n_{1}(1 ; \beta, \zeta)$ is analytic for all interior points of the arc $A_{1} A_{2}$ as well as for all points not on the unit circle.

An extension of this method serves to prove the corresponding theorems for the two-body distribution function $n_{2}(1,2 ; \beta, \zeta)$. Generalizing (44), we may write

so that

$$
\begin{aligned}
\Xi\left(\beta, \zeta_{1}, \zeta_{2}, \zeta, \ldots, \zeta ; V\right)= & P^{\prime}(\zeta, V)+\zeta_{1} Q_{1}^{\prime}(\zeta, V) \\
& +\zeta_{2} Q_{2}^{\prime}(\zeta, V)+\zeta_{1} \zeta_{2} R^{\prime}(\zeta, V)
\end{aligned}
$$

$$
n_{2}(1,2 ; \beta, \zeta ; V)=\zeta^{2} R^{\prime} /\left[P^{\prime}+\zeta\left(Q_{1}^{\prime}+Q_{2}^{\prime}\right)+\zeta^{2} R^{\prime}\right]
$$

The analogue of $(45)$ [or (54)] is now

where

$$
\frac{n_{2}(1,2 ; \beta, \zeta ; V)}{1-n_{1}(1 ; \beta, \zeta ; V)-n_{1}(2 ; \beta, \zeta ; V)+n_{2}(1,2 ; \beta, \zeta ; V)}=f^{\prime}(\zeta, V)
$$

$$
f^{\prime}(\zeta, V)=\zeta^{2} R^{\prime}(\zeta, V) / P^{\prime}(\zeta, V)
$$

Thus $n_{2}$ can be calculated if $f^{\prime}$ and the one-particle distribution function are known.

Let $\xi_{1}(\zeta, V)$ and $\xi_{2}(\zeta, V)$ be the roots of the quadratic equation

$$
P^{\prime}(\zeta, V)+\left[Q_{1}^{\prime}(\zeta, V)+Q_{2}^{\prime}(\zeta, V)\right] \xi+R^{\prime}(\zeta, V) \xi^{2}=0
$$


so that (64) implies

$$
f^{\prime}(\zeta, V)=\zeta^{2} / \xi_{1}(\zeta) \xi_{2}(\zeta)
$$

For ferromagnetic interactions, Lee and Yang's lemma implies that if $\Xi(\beta ; \xi, \xi, \zeta, \ldots, \zeta ; V)=0$ and $|\zeta| \geqq 1$ then $|\xi| \leqq 1$; it follows by $(66)$ that

$$
\left|f^{\prime}(\zeta, V) / \zeta^{2}\right|\{\gtreqless 1 \text { if }|\zeta|\{\gtreqless\} 1 \text {. }
$$

Moreover, if the point $f^{\prime}(\zeta, V)$ moves twice around the unit circle, then (66) shows that at least one of the numbers $\zeta / \xi_{1}$ and $\zeta / \xi_{2}$ must take the value 1 . Since $\Xi(\beta, \zeta ; V)=0$ [by $(61)$ and $(65)]$ if $\zeta=\xi_{1}$ or $\xi_{2}$, but has no zeros on $A_{1} A_{2}$ for large $V$, we see that $\arg f^{\prime}(\zeta, V)$ cannot increase by as much as $4 \pi$ when $\zeta$ traverses the arc $A_{1} A_{2}$. Consequently, by a similar argument to the one used previously, the number of zeros of $f^{\prime}(\zeta, V)$ that lie inside $G^{\prime}$ is at most 1 . If this number is actually 0 for all sufficiently large $V$, then the proof goes through as before and we conclude that $f^{\prime}(\zeta)$ exists and is analytic in $G$. If the number is 1 and the set of zeros of $f^{\prime}(\zeta, V)$ has, as $V \rightarrow \infty$, a limit point $q$ within $G^{\prime}$ then, by Hurwitz' theorem, this limit point is also a zero of $f^{\prime}(\zeta)$ and the proof again goes through as before provided we exclude a neighborhood of $q^{\prime}$ from $G^{\prime}$ and the inversion of this neighborhood from $G^{\prime \prime}$. Finally, if $f^{\prime}(\zeta)$ has no zero in $G^{\prime}$ but some or all of the functions $f^{\prime}(\zeta, V)$ have zeros in $G^{\prime}$, then for large $V$ any zero of $f^{\prime}(\zeta, V)$ in $G^{\prime}$ must be very close to the boundary of $G^{\prime}$. A single zero chose to the straight boundary does not materially affect our proof, and a single zero very close to the arc $A_{1} A_{2}$ contributes a factor that tends to 1 in the limit $V \rightarrow \infty$ and therefore does not affect the value of $f^{\prime}(\zeta)$. Thus in all cases we conclude that $f^{\prime}(\zeta)$ is analytic in $G$, with the possible exception of a simple pole in $G^{\prime \prime}$; consequently, by $(63), n_{2}(1,2 ; \beta, \zeta)$ also exists for $|\zeta| \neq 1$ and on the $\operatorname{arc} A_{1} A_{2}$, and is a meromorphic function of $\zeta$.

In a similar way we can prove that $n_{3}(1,2,3 ; \beta, \zeta)$ exists and is meromorphic in $\zeta$, then $n_{4}$, and so on. Finally, by applying the lemma of Section III, we conclude that every distribution function $n_{s}\left(\boldsymbol{X}_{s} ; \beta, \zeta\right)$ is analytic in both $\beta$ and $\zeta$ provided $\beta>0$ and $\zeta$ is neither a pole of $n_{s}\left(\boldsymbol{X}_{s} ; \beta, \zeta\right)$ nor a limit point of zeros of $\Xi$.

\section{Analytic Properties of Distribution Functions: Non-negative Potentials}

The second type of system for which we can usefully apply Vitali's theorem to the distribution functions is a system with non-negative potential, satisfying $\varphi(r) \geqq 0$ for all $r$. Here the region $J$ within which we can prove analyticity in $z$ at fixed $\beta$ is the $\operatorname{disk}|z|<\lim _{V \rightarrow \infty} R(\beta, V)$ where $R(\beta, V)$ is the distance from the origin of the $z$-plane to the nearest zero of $\Xi(\beta, z ; V)$. We choose $K$ to be any subset of $J$ that is 
the closure of an open set; then $K$ is free of zeros of $\Xi(\beta, z ; V)$ for all sufficiently large $V$.

It is convenient here to work with the Ursell functions, defined in (2), rather than the distribution functions. For a finite volume, the Ursell functions have the power series expansions [18]

$$
\begin{aligned}
u_{s}\left(\boldsymbol{X}_{s} ; \beta, z ; V\right)= & \sum_{n=0}^{\infty} \frac{z^{s+n}}{n !} \int_{V} \cdots \int_{V} U_{s+n} \\
& \cdot\left(\boldsymbol{x}_{1}, \ldots, \boldsymbol{x}_{s+n} ; \beta\right) d \boldsymbol{x}_{s+1} \ldots d \boldsymbol{x}_{s+n}
\end{aligned}
$$

where $U_{l}\left(\boldsymbol{x}_{1}, \ldots, \boldsymbol{x}_{l} ; \beta\right)$ denotes the $l$-body cluster function defined for $l=1$ by $U_{1}\left(x_{1} ; \beta\right)=1$ and for larger values of $l$ by

$$
U_{l}\left(\boldsymbol{x}_{1}, \ldots, \boldsymbol{x}_{l} ; \beta\right)=\sum_{\Gamma} \prod_{(i j) \in \Gamma}\left[e^{-\beta \varphi\left(\left|\boldsymbol{x}_{i}-\boldsymbol{x}_{l}\right|\right)}-1\right]
$$

with the sum going over all connected graphs with vertices labelled $1,2, \ldots, l$. The series (68) converges absolutely for all $z$ in $K$ and all sufficiently large $V$, since $u_{s}\left(\boldsymbol{X}_{s} ; \beta, z ; V\right)$, being defined in terms of distribution functions, is singular only at the zeros of $\boldsymbol{\Xi}(\beta, z ; V)$. We may therefore integrate term by term with respect to $\boldsymbol{x}_{2}, \ldots, \boldsymbol{x}_{s}$; if we then take absolute values on both sides, we obtain the inequality

$$
\begin{aligned}
&\left|\int_{V} \cdots \int_{V} u_{s}\left(\boldsymbol{X}_{s} ; \beta, z ; V\right) d \boldsymbol{x}_{2} \ldots d \boldsymbol{x}_{s}\right| \\
& \leqq \sum_{n=0}^{\infty} \frac{|z|^{s+n}}{n !} \int \cdots \int\left|U_{s+n}\left(\boldsymbol{X}_{s+n} ; \beta\right)\right| d \boldsymbol{x}_{2} \ldots d \boldsymbol{x}_{s+n}
\end{aligned}
$$

where the integrals on the right range over all space.

Groeneveld [1] has shown that

$$
U_{l}\left(\boldsymbol{X}_{l}\right)=(-1)^{l+1}\left|U_{l}\left(\boldsymbol{X}_{l}\right)\right|
$$

for non-negative potentials with $\beta>0$. Substituting this into (70) and using the series (4) in the form

$$
\beta p(\beta, z)=z+\sum_{l=2}^{\infty} \frac{z^{l}}{l !} \int \cdots \int U_{l}\left(\boldsymbol{X}_{l} ; \beta\right) d \boldsymbol{x}_{2} \ldots d \boldsymbol{x}_{l}
$$

we obtain

$$
\left|\int_{V} \cdots \int_{V} u_{s}\left(\boldsymbol{X}_{s} ; \beta, z\right) d \boldsymbol{x}_{2} \ldots d \boldsymbol{x}_{s}\right| \leqq\left|z_{1}^{s}\left(\partial / \partial z_{1}\right)^{s}\left[\beta p\left(\beta, z_{1}\right)\right]\right|_{z_{1}=-|z|} \text {. }
$$

By Yang and Lee's theorem, the function $z^{s}(\partial / \partial z)^{s}(\beta p)$ is analytic in $z$, and therefore bounded, for all $z$ in $K$; hence the left side is also bounded for all $z$ in $K$ and all sufficiently large $V$. In a similar way we can show that

$$
|\underbrace{\int \cdots \int}_{\omega} u_{s}\left(\boldsymbol{X}_{s} ; \beta, z ; V\right) d \boldsymbol{x}_{2} \ldots d \boldsymbol{x}_{s}|
$$


where the multiple integral ranges over an arbitrarily small region $\omega$ in $\left(\boldsymbol{x}_{2}, \ldots, \boldsymbol{x}_{s}\right)$-space, is bounded for all $z$ in $K$ and all sufficiently large $V$. For a lattice system, the integrals in (73) are replaced by sums, and the expression (74) by the absolute value of any individual term in this sum.

It follows by Vitali's theorem that for non-negative potentials with $\beta>0$ the integral on the left of (73), the integral $(74)^{3}$ and, in the case of a lattice system, $u_{s}\left(\boldsymbol{X}_{s} ; \beta, z\right)$ and $n_{s}\left(\boldsymbol{X}_{s} ; \beta, z\right)$ themselves, are analytic in $z$ at constant $\beta$ and $\boldsymbol{X}_{s}$. That they are, in fact, analytic in both $z$ and $\beta$ now follows as before by the methods of section IV, provided only that $J$ is redefined to be

$$
|z|<\inf _{\beta \in I} R(\beta) \text { with } R(\beta) \equiv \lim _{V \rightarrow \infty} R(\beta, V),
$$

so that Vitali's theorem applies to the same $z$-plane region $K$ for all $\beta$ in $I$. For these potentials, $R(\beta)$ is [3] the radius of convergence of the Mayer series (4).

\section{The Cluster Property: Ising Ferromagnet}

Having shown that the distribution function $n_{s}\left(\boldsymbol{X}_{s} ; \beta, z\right)$ and the corresponding Ursell functions $u_{s}$ are analytic in $\beta$ and $z$ at fixed $\boldsymbol{X}_{s} \equiv\left(\boldsymbol{x}_{1}, \ldots, \boldsymbol{x}_{s}\right)$ under suitable conditions, we now consider the nature of their dependence on the position vectors $\boldsymbol{x}_{1}, \ldots, \boldsymbol{x}_{s}$ at fixed $\beta, z$ under these same conditions. The general property we wish to demonstrate is that $u_{s}\left(\boldsymbol{X}_{s} ; \beta, z\right)$ is small when the points $\boldsymbol{x}_{1}, \ldots, \boldsymbol{x}_{s}$ are widely separated. The natural measure of the wideness of this separation is the diameter $\Delta_{s}$ of the point set $\boldsymbol{X}_{s}$,

$$
\Delta_{s}\left(\boldsymbol{X}_{s}\right) \equiv \max _{i, j \leqq s}\left|\boldsymbol{x}_{i}-\boldsymbol{x}_{j}\right| \text {. }
$$

For lattice systems with $\varphi(r) \leqq 0$ for $r>0$ (such as the Ising ferromagnet), we shall show that, for suitable values of $\beta$ and $z$,

$$
\lim _{\Delta_{s}\left(\boldsymbol{X}_{s}\right) \rightarrow \infty} u_{s}\left(\boldsymbol{X}_{s}\right)=0
$$

by which we mean that we can find a function $\eta(\Delta)$ with the properties

and

$$
\lim _{\Delta \rightarrow \infty} \eta(\Delta)=0
$$

$$
\left|u_{s}\left(\boldsymbol{X}_{s} ; \beta, z\right)\right| \leqq \eta\left[\Delta_{s}\left(\boldsymbol{X}_{s}\right)\right]
$$

for all $\boldsymbol{X}_{s}$. If the potential $\varphi(r)$ is cut off (i.e., if it vanishes for all sufficiently large $r$ ), we shall show that $\eta(\Delta)$ can be a decreasing exponential, and then it follows that the Ursell functions also have the cluster property

3 The stronger result that, in the continuum case, $u_{s}, n_{s}$ and even $\log \left(n_{s} / z^{8}\right)$ are analytic in $z$ for $|z|<R(\beta)$ has been obtained by Groeneveld [17]. 
(3). For non-negative potentials, we shall prove (3) directly but we have not been able to prove (76).

We consider first the case of an Ising ferromagnet. As before, we use the variable $\zeta$ in the place of $z$, and assume that $|\zeta|<1$ and $\beta>0$, so that the expansion (68) converges and may be written in the form

where

$$
u_{s}\left(\boldsymbol{X}_{s} ; \beta, \zeta ; V\right)=\sum_{n=0}^{\infty} \frac{\zeta^{n+s}}{n !} \sum_{\boldsymbol{x}_{s+1} \in V} \cdots \sum_{\boldsymbol{x}_{s+n} \in V} U_{s+n}^{\prime}\left(\boldsymbol{X}_{s+n} ; \beta\right)
$$

with

$$
U_{l}^{\prime}\left(\boldsymbol{X}_{l}\right) \equiv U_{l}\left(\boldsymbol{X}_{l}\right) \exp \left[-\beta \sum_{i=1}^{l} \alpha\left(\boldsymbol{x}_{i}\right)\right]
$$

$$
\alpha(\boldsymbol{x}) \equiv-\frac{1}{2} \sum_{\boldsymbol{y} \in V \text { and } \neq \boldsymbol{x}} \varphi(|\boldsymbol{y}-\boldsymbol{x}|) .
$$

By writing (62) in the form $n_{2}=\zeta^{2} /\left[\zeta-\xi_{1}(\zeta)\right]\left[\zeta-\xi_{2}(\zeta)\right]$, and using the fact (obtained from Lee and Yang's lemma, as in the discussion of (66)) that if $|\zeta| \leqq 1$ then $|\xi| \geqq 1$, we see that $\left|n_{2}\right| \leqq|\zeta|^{2} /(1-|\zeta|)^{2}$ if $|\zeta| \leqq 1$. Using this and its generalization $n_{s} \leqq|\zeta|^{s}(1-|\zeta|)^{-s}$ in the definitions (2) of the Ursell functions, we obtain the upper bound

$$
\left|u_{s}\left(\boldsymbol{X}_{s} ; \beta, \zeta ; V\right)\right| \leqq c_{s}|\zeta|^{s} /(1-|\zeta|)^{s} \quad \text { if } \quad|\zeta|<1, \quad \beta>0
$$

where $c_{s}$ is the sum of the absolute values of the coefficients on the right of the $s$ th equation of the set (2). Applying Cauchy's inequalities to the function $u_{s} \mid \zeta^{s}$, using the contour $|\zeta|=a=$ const., we obtain, by (78) and (81), the bound

$$
\left|\sum_{\boldsymbol{x}_{s+1} \in V} \cdots \sum_{\boldsymbol{x}_{s+n} \in V} U_{s+n}^{\prime}\left(\boldsymbol{X}_{s+n} ; \beta\right)\right| \leqq n ! c_{s} a^{-n}(1-a)^{-s}
$$

which holds for any non-negative integer $n$ and any $a$ between 0 and 1 .

If we choose $a$ in the range $|\zeta|<a<1$, then (82) shows that the series (78) is majorized by a convergent geometric series. From this fact it follows, by Weierstrass' test, that at fixed $\zeta$ the series (78) converges uniformly with respect to the variables $V$ and $\boldsymbol{X}_{s}$, so that limiting operations affecting these variables may be carried out term by term. To prove (76), therefore, we need only show that each term of the series tends to zero in the double limit where first $V \rightarrow \infty$ and then $\Delta \rightarrow \infty$. Since the limit $V \rightarrow \infty$ is trivial, it is a question of proving that, for every value of $n$,

Since

$$
\lim _{\Delta \rightarrow \infty} \sum_{\boldsymbol{x}_{s+1}} \cdots \sum_{\boldsymbol{x}_{s+n}} U_{s+n}\left(\boldsymbol{X}_{s+n} ; \beta\right)=0
$$

$$
\left|\sum_{\boldsymbol{x}_{s+1}} \cdots \sum_{\boldsymbol{x}_{s+n}} U\right| \leqq \sum_{\boldsymbol{x}_{s+1}} \cdots \sum_{\boldsymbol{x}_{s+n}}|U| \leqq \sum_{\boldsymbol{x}_{3}} \cdots \sum_{\boldsymbol{x}_{s+n}}|U|
$$


and $\Delta\left(\boldsymbol{x}_{1}, \ldots, \boldsymbol{x}_{s}\right) \geqq x_{12} \equiv\left|\boldsymbol{x}_{1}-\boldsymbol{x}_{2}\right|$, a sufficient condition for (83) to hold is

$$
\lim _{\boldsymbol{x}_{12} \rightarrow \infty} \sum_{\boldsymbol{x}_{3}} \cdots \sum_{\boldsymbol{x}_{s+n}}\left|U_{s+n}\left(\mathbf{X}_{s+n} ; \beta\right)\right|=0 .
$$

Our proof of (85) depends on the Ursell-Mayer formula (69). Writing $f_{i j}$ or $f\left(x_{i j}\right)$ for the square bracket in (69) and substituting into (85), we see that it is sufficient to prove

$$
\sum_{\boldsymbol{x}_{3}} \cdots \sum_{\boldsymbol{x}_{s+n}} \prod_{(i j) \in \Gamma}\left|f_{i j}\right| \rightarrow 0 \text { as } \quad x_{12} \rightarrow \infty
$$

for every graph summed over in (69). Since $\Gamma$ is connected, it can be converted into a Cayley tree by deleting some bonds. Let $T$ denote this Cayley tree (it is not unique) and $T^{\prime}$ the part of $T$ that joins vertices 1 and 2 . An upper bound on the sum (86) is then given by

$$
\begin{aligned}
& \left(f_{\max }\right)^{k} \sum_{\boldsymbol{x}_{3}} \cdots \sum_{\boldsymbol{x}_{s+n}} \prod_{(i j) \in T}\left|f_{i j}\right| \\
& =\left(f_{\max }\right)^{k}[B(\beta)]^{s+n-m-1} \sum_{\boldsymbol{x}_{3}} \cdots \sum_{\boldsymbol{x}_{m+1}} \prod_{(i j) \in T^{\prime}}\left|f_{i j}\right|
\end{aligned}
$$

where $k$ is the number of bonds that are in $\Gamma$ but not in $T, m$ is the number of bonds in $T^{\prime}$ and we have relabelled the variables of summation and the corresponding vertices of $T$, so that the bonds in $T^{\prime}$ become $(1,3),(3,4), \ldots,(m, m+1),(m+1,2)-$ or just $(1,2)$ if $m=1$. We have also used the definition (9), which might be written $B(\beta)=\sum_{\boldsymbol{x}}\left|f_{\boldsymbol{x}}\right|$ in the notation of this section. We divide the summation in the last line of (87) into $m$ parts, say $\sum_{13}, \sum_{34}, \ldots, \sum_{m+1,2}$ such that $\sum_{r s}=0$ only if $x_{r s}$ is at least as great as any of the other distances in the chain $x_{13}$, $x_{34}, \ldots, x_{m+1,2}$. In $\sum_{13}$, since $x_{13}+x_{34}+\cdots+x_{m+1,2} \geqq x_{12}$, we have $x_{13} \geqq x_{12} / m$ and therefore

where

$$
\begin{gathered}
\sum_{13} \leqq \sum_{\boldsymbol{x}_{3}} \cdots \sum_{\boldsymbol{x}_{m+1}} f_{\max }\left(x_{12} / m\right)\left|f_{34} f_{45} \cdots f_{m+1,2}\right| \\
=[B(\beta)]^{m-1} f_{\max }\left(x_{12} / m\right)
\end{gathered}
$$

$$
f_{\max }(y)=\sup _{x \geqq y}|f(x)| .
$$

It follows from the convergence of the sum for $B(\beta)$ that $f(x)$ tends to 0 for $x \rightarrow \infty$. Consequently by (88) we find that $\sum_{13} \rightarrow 0$ for $x_{12} \rightarrow \infty$ and hence, since similar arguments apply to $\sum_{34}, \sum_{45}$, etc., that the expression (86) does the same. This proves (85) and hence completes the proof of (76).

In the special case of a cut-off potential, that is, one where

$$
\varphi(r)=0 \quad \text { if } \quad r>\lambda
$$


for some $\lambda$ (the range of the potential), the function $\eta(\Delta)$ in (77) takes a simple form. Equation (88) now shows that $\sum_{13}=0$ if $x_{12} / m>\lambda$, and working back to (83) we find (since $m \leqq s+n-1$ ) that

$$
\sum_{\boldsymbol{x}_{s+1}} \cdots \sum_{\boldsymbol{x}_{s+n}} U_{s+n}\left(\boldsymbol{X}_{s+n} ; \beta\right)=0 \text { if } \Delta\left(\boldsymbol{X}_{s}\right)>(s+n-1) \lambda .
$$

The series analogous to $(78)$ for $u_{s}\left(\boldsymbol{X}_{s} ; \beta, \zeta\right)$ thus begins at the term in $\zeta^{s+n\left(\boldsymbol{X}_{s}\right)}$ where $n\left(\boldsymbol{X}_{s}\right)$ is the smallest integer satisfying

$$
\Delta\left(\boldsymbol{X}_{s}\right) \leqq\left[s+n\left(\boldsymbol{X}_{s}\right)-1\right] \lambda .
$$

Using the majorization (82) in this series we obtain

$$
\left|u_{s}\left(\boldsymbol{X}_{s} ; \beta, \zeta\right)\right| \leqq \frac{c_{s}}{1-|\zeta| / a}\left(\frac{a}{1-a}\right)^{s}\left(\frac{|\zeta|}{a}\right)^{1+\Delta\left(\boldsymbol{x}_{s}\right) / \lambda} .
$$

for $|\zeta|<a<1$. For example, we can study the spin-spin correlation function $u_{2}(\boldsymbol{r})$ for an Ising ferromagnet in field $H$ by choosing $s=2$ and $\boldsymbol{x}_{2}=\boldsymbol{x}_{1}+\boldsymbol{r}$, so that $\Delta=r$; equations (92) and (37) then show that $u_{2}(\boldsymbol{r})$ falls off with distance according to

$$
\left|u_{2}(\boldsymbol{r})\right|<\text { const. } \exp [(-2 m|\operatorname{Re} H \beta|+\varepsilon) r / \lambda]
$$

where $\varepsilon=\log (1 / \alpha)$ is any small positive number, and the constant may depend on $H$ and $\varepsilon$.

The exponentially decaying upper bound (92) also has the consequence that the cluster property $(3)$ holds when $|\zeta|<1$ or (by symmetry) $|\zeta|>1$ for a lattice gas with a cut-off attractive potential.

\section{The Cluster Property for Non-negative Potentials}

For non-negative potentials we can prove the cluster property both for continuum and lattice systems, provided again that the conditions

$$
\beta>0 \text { and }|z|<R(\beta)
$$

are satisfied. For the lattice system, the proof depends on the result obtained in $\S$ VI, that $u_{s}\left(\boldsymbol{X}_{s} ; \beta, z\right)$ is analytic in $z$ if $(94)$ holds. From this result it follows that the series expansion analogous to (68), namely

$$
u_{s}\left(\boldsymbol{X}_{s} ; \beta, z\right)=\sum_{n=0}^{\infty} \frac{z^{s+n}}{n !} \sum_{\boldsymbol{x}_{s+1}} \cdots \sum_{\boldsymbol{x}_{s+n}} U_{s+n}\left(\boldsymbol{X}_{s+n} ; \beta\right)
$$

(where the sums range over an infinite lattice), converges when (94) holds; hence, taking absolute values and summing over $\boldsymbol{x}_{2}, \ldots, \boldsymbol{x}_{s}$, we obtain

$$
\sum_{\boldsymbol{x}_{2}} \cdots \sum_{\boldsymbol{x}_{s}}\left|u_{s}\left(\boldsymbol{X}_{s} ; \beta, z\right)\right| \leqq \sum_{n=0}^{\infty} \frac{|z|^{s+n}}{n !} \sum_{\boldsymbol{x}_{2}} \cdots \sum_{\boldsymbol{x}_{s+n}}\left|U_{s+n}\left(\boldsymbol{X}_{s+n} ; \beta\right)\right| \text {. }
$$

The interchange of summations is valid since all terms in the series are positive. As shown in the derivation of (73), the right side of (96) is equal 
to the value taken by the function $-z_{1}^{s}\left(\partial / \partial z_{1}\right)^{s}\left[\beta p\left(\beta, z_{1}\right)\right]$ when $z_{1}=-|z|$. By (94), this function is analytic, and therefore finite, when $z_{1}=-|z|$; thus (96) implies that the Ursell functions have the cluster property (3).

A similar argument applies to continuum systems; this time we start from two functions defined by the formula (74) with $\omega$ taken, for one of them, to be the part of $\left(\boldsymbol{x}_{2}, \ldots, \boldsymbol{x}_{s}\right)$-space (call it $\left.\omega^{+}\right)$where

$$
u_{s}\left(\boldsymbol{x}_{1}, \ldots, \boldsymbol{x}_{s} ; \beta, z\right)>0
$$

and for the other, the part (call it $\omega^{-}$) where $u_{s}<0$. As shown in $\S$ VI, both these functions are analytic in $z$ at fixed $\boldsymbol{x}_{1}, \omega^{+}$and $\beta$ when (94) holds; therefore (95) has the analogue

$$
\begin{aligned}
& \underbrace{\int \cdots \int}_{\omega^{+}} u_{s}\left(\boldsymbol{X}_{s} ; \beta, z\right) d \boldsymbol{x}_{2} \ldots d \boldsymbol{x}_{s}=\sum_{n=0}^{\infty} \frac{z^{n+s}}{n !} \underbrace{\int \cdots \int}_{\omega^{+}} \\
& \int \cdots \int U_{s+n}\left(\boldsymbol{X}_{s+n} ; \beta\right) d \boldsymbol{x}_{2} \ldots d \boldsymbol{x}_{s+n}
\end{aligned}
$$

where the first $s-1$ integrations on the right are over $\omega^{+}$and the remaining $n$ are over all space. Combining (97) with the corresponding result for $\omega^{-}$we obtain

$$
\begin{aligned}
& \int \ldots \int\left|u_{s}\left(\boldsymbol{X}_{s} ; \beta, z\right)\right| d \boldsymbol{x}_{2} \ldots d \boldsymbol{x}_{s} \leqq \sum_{n=0}^{\infty} \frac{|z|^{n+s}}{n !} \int \cdots \int \\
& \cdot\left|U_{s+n}\left(\boldsymbol{X}_{s+n} ; \beta\right)\right| d \boldsymbol{x}_{2} \ldots d \boldsymbol{x}_{s+n}
\end{aligned}
$$

where the integrations now range over all space. Just as in the discussion of (96) it follows that the cluster property (3) holds for continuum systems with non-negative potentials, under the condition (94).

For a potential with a cut-off, we can show (as in VII) that the convergence constituting the cluster property (3) is exponential. That is to say, if $k$ is any positive integer $\geqq s$, the contribution to the sum on the left of (96) from configurations $\boldsymbol{X}_{s}$ where $\Delta\left(\boldsymbol{X}_{s}\right)$ exceeds $k \lambda$ must fall away exponentially with increasing $k$, and similarly for the integral on the left of (98). To show this for lattice systems, we retrace the derivation of $(96)$, but instead of summing over all $\boldsymbol{x}_{2}, \ldots, \boldsymbol{x}_{s}$, we restrict the summation to those terms for which $\Lambda\left(\boldsymbol{X}_{s}\right)>k \lambda$. Then by $(91)$, the terms for which $s+n-1 \leqq k$ in the sum on the right will vanish. Using Cauchy's inequality applied to the $s$ th derivative of the power series (72) we can estimate the remaining terms of this sum, obtaining

$$
\begin{aligned}
& \underbrace{\sum_{\Delta\left(\boldsymbol{X}_{s}\right)>k \lambda} \sum_{\boldsymbol{x}_{3}}}_{\boldsymbol{x}_{2}}\left|u_{s}\left(\boldsymbol{X}_{s} ; \beta, z\right)\right| \leqq \sum_{n=k+2}^{\infty} \frac{|z|^{s+n}}{n !} \frac{n ! M_{s}(\beta, a)}{a^{n}} \\
= & \frac{a^{s} M_{s}(\beta, a)}{1-|z| \mid a}\left(\frac{|z|}{a}\right)^{k+2}
\end{aligned}
$$


where $M_{s}(\beta, a)$ is the maximum absolute value of the function $\left(\partial / \partial z_{1}\right)^{s}$ - $\left[\beta p\left(\beta, z_{1}\right)\right]$ on any circle $\left|z_{1}\right|=a$ whose radius satisfies

$$
|z|<a<R(\beta) \text {. }
$$

Since every term in the sum on the left side of (99) is positive, its right side also provides a bound, similar to the one obtained in (92), on the Ursell functions themselves:

$$
\left|u_{s}\left(\boldsymbol{X}_{s} ; \beta, z\right)\right| \leqq \frac{a^{s} M_{s}(\beta, a)}{1-|z| \mid a}\left(\frac{|z|}{a}\right)^{1+\Delta\left(\boldsymbol{X}_{s}\right) / \lambda} .
$$

Using the isomorphism pointed out by LEE and YANG [10], we may apply the results (99) and (101) to the Ising antiferromagnet. These results show, for example, that the spin-spin correlation function $u_{2}(\boldsymbol{v})$ falls off with distance according to

if

$$
\left|u_{2}(\boldsymbol{r})\right|<\text { const. } \exp [(-2 \beta m[|\operatorname{Re} H|-H(\beta)]+\varepsilon) r / \lambda]
$$

$$
\beta>0 \quad \text { and } \quad|\operatorname{Re} H|>H(\beta) \equiv \frac{1}{2 m \beta}\left[\log \frac{1}{R(\beta)}-\alpha \beta\right] .
$$

The quantity we have called $H(\beta)$ vanishes for a ferromagnet because of Lee and Yang's theorem, but for an antiferromagnet it is presumably positive, so that the result (102) is weaker than its counterpart (93) for the ferromagnet.

For a continuum system whose potential has a cut-off, the integral analogue of (99) still holds, (generalizing a result given by RUELLE [4]) but we have not been able to prove the analogue of (101).

\section{Discussion}

Our main results fall into two categories. First, there are the proofs based on the lemma of Section III, showing that if the function under consideration is analytic in a region $J$ of the $z$-plane for all $\beta$ in an interval $I$ of the real axis, then it is in fact analytic in both $\beta$ and $z$. Secondly, there are the proofs, based on Vitali's theorem, showing that in the thermodynamic limit these functions actually are analytic in $z$ for some region of the $z$ plane, and that they satisfy the cluster property.

The results in the first category are very general; the only further generalization that might be possible is to eliminate the condition that $J$ must include the origin of the $z$-plane, which restricts the theory to gaseous or fluid phases. The results in the second category refer to particular types of systems and are much less complete. Our best set of results in this category refers to the Ising ferromagnet. Here we have shown that the thermodynamic functions and distribution functions are analytic in both $\beta$ and $\zeta$ if $\beta>0$ and $\zeta$ is not a limit point of zeros of the grand partition function (and in particular if $|\zeta| \neq 1$ ). Thus if we 
know there is a singularity, say at $\beta=\beta_{c}$, in the thermal properties at zero magnetic field (for example the logarithmic singularity, discovered by ONSAGER, in the specific heat of a two-dimensional ferromagnet) we can now deduce without knowing anything about the magnetic properties of the system that the zeros of $\Xi$ in the $\zeta$ plane have a limit point at $\zeta=1$ when $\beta=\beta_{c}$. We have also shown for the general Ising ferromagnet that if $|\zeta| \neq 1$ then the $s$-particle Ursell functions tend to zero as $\Delta \rightarrow \infty$ and that this decay is rapid enough to give the cluster property (3) if the potential has a cut-off; to complete this set of results it would be desirable to prove the cluster property for values of $\zeta$ on the unit circle that are not limit points of zeros and to remove the restriction to cut-off potentials. For values of $\zeta$ that are limit points of zeros, even the existence of $n_{s}$ has not been proved, apart from the case $\zeta=1$, where it is proved for $s=1,2$ by Griffiths [19] and where it follows for $s \geqq 3$ from Kelly and Sherman's generalization [20] of Griffiths' work.

The only other systems for which results of a similar degree of completeness are possible at present are one-dimensional. For the onedimensional fluid with nearest-neighbor interactions, for example, one can show by an extension of the methods used by Elvey and Penrose [21] that (at fixed $\beta$ ) the distribution functions are analytic in $z$, and have the cluster property, for all values of $z$ where $p(\beta, z)$ is analytic in $z$; analyticity in both variables then follows by the lemma of Section IIII. In general, however, it may not be possible to deduce analyticity of distribution functions from that of thermodynamic functions alone, even in the Ising ferromagnet. Our proof for the distribution functions with $|\zeta|=1$ requires the arc $A_{1} A_{2}$ to be free of zeros for large $V$, whereas analyticity of the thermodynamic functions implies only that the number of zeros in any sub-arc of $A_{1} A_{2}$ is $o(V)$ as $V \rightarrow \infty$.

There is an alternative method of investigating the existence and properties of correlation functions, which brings out the connection between the two categories of results. (A similar method was used by Fisher [22], but he proves only convexity in $\lambda$, not analyticity.) Suppose that the system is placed in an external potential

$$
\Phi(\boldsymbol{x}) \equiv-\left[\lambda_{1} \psi_{1}(\boldsymbol{x})+\lambda_{2} \psi_{2}(\boldsymbol{x})+\cdots+\lambda_{s} \psi_{s}(\boldsymbol{x})\right] k T
$$

where $\lambda_{1}, \ldots, \lambda_{s}$ are new complex variables and $\psi_{1}(\boldsymbol{x}), \ldots, \psi_{s}(\boldsymbol{x})$ are periodic functions of $\boldsymbol{x}$, with an arbitrary unit cell of volume $\Gamma$ which is the same for all the $s$ functions. The grand partition function for a system in this situation has the property

$$
\begin{aligned}
{\left[\frac{\partial^{s}}{\partial \lambda_{1} \ldots \partial \lambda_{s}}-\frac{\log \Xi}{V}\right]_{\lambda_{1}=\cdots=\lambda_{s}=0}=\frac{1}{V} } \\
\cdot \int_{V} d \boldsymbol{x}_{1} \ldots \int_{V} d \boldsymbol{x}_{s} \psi_{1}\left(\boldsymbol{x}_{1}\right) \ldots \psi_{s}\left(\boldsymbol{x}_{s}\right) \hat{u}_{s}\left(\boldsymbol{X}_{s} ; \beta, z ; V\right) .
\end{aligned}
$$


Applying the lemma of Section III with $\lambda_{i}$ replacing $\beta$, we can show, under suitable conditions, that the infinite-volume limit of this equation is

$$
\begin{aligned}
\left.\frac{\partial^{s}(\beta p)}{\partial \lambda_{1} \ldots \partial \lambda_{s}}\right|_{\lambda_{1}=} & =\lambda_{s}=0 \\
& =\frac{1}{T} \\
& \int_{\Gamma} d \boldsymbol{x}_{1} \int d \boldsymbol{x}_{2} \ldots \int d \boldsymbol{x}_{s} \psi_{1}\left(\boldsymbol{x}_{1}\right) \ldots \psi_{s}\left(\boldsymbol{x}_{s}\right) \hat{u}_{s}\left(\boldsymbol{X}_{s} ; \beta, z\right)
\end{aligned}
$$

where the infinite-volume modified Ursell function ${ }^{4} \hat{u}_{s}\left(\boldsymbol{X}_{s} ; \beta, z\right)$ is now defined as the function that makes (105) true for all members of a sufficiently wide class of test functions $\psi_{i}(\boldsymbol{x})$, with $\beta p$ the infinite volume limit of $\log \Xi / V$.

In order to prove the existence of $u_{s}\left(\boldsymbol{X}_{s}\right)$ by this method, however, it is necessary to show that if $\left|\lambda_{1}\right|, \ldots,\left|\lambda_{s}\right|$ are small then the zeros of $\Xi\left(\beta, z ; \lambda_{1}, \ldots, \lambda_{s} ; V\right)$ do not penetrate far into the $z$-plane region $J$ defined in (36). At the time of writing we have been able to do this only for the two types of system discussed in Sections V-VIII, the Ising ferromagnet and systems with non-negative potentials; so this alternative method, though pleasing because it makes the theorem of Section III a unifying principle for the whole discussion, has not so far yielded any additional results.

Acknowledgements. The authors would like to thank R. B. Griffiths and G. A. Baker, Jr., for helpful discussions, and R. L. Dobrushin, G. Gallavottr, S. Mtracle-Sole and J. Groeneveld for advance information about their work. Most of all, we are indebted to D. RuelLe for showing us how to formulate and prove the lemma of $\S$ III, and to him and G. GaLLAvotTI for helpful criticisms of the typescript.

\section{References}

1. Groeneveld, J.: Phys. Letters 3, 50 (1962).

2. Ruelle, D.: Ann. Phys. 25, 109 (1963).

3. Penrose, O.: J. Math. Phys. 4, 1312 (1963).

4. Ruelle, D.: Rev. Mod. Phys. 36, 580 (1964).

5. Uhlenbeck, G. E., and G. W. Ford: In: Studies in statistical mechanics, Vol. 1 (ed. De Boer and Uhlenbeck) pp. 143 and 137, equations (46) and (29). Amsterdam: North Holland Publishing Co. 1962.

6. Ahlfors, L. A.: Complex analysis, p. 24. New York: McGraw-Hill 1966.

7. Dobrushin, R. L.: Teor. Veroyatnostei i ee primeneniya 9, 626 (1964); Theory Probability. Appl. USSR 9, 566 (1964).

8. Jones, G. L.: J. Math. Phys. 7, 2000 (1966).

9. Smith, E. B., and B. J. Alder: J. Chem. Phys. 30, 1190 (1959).

10. LeE, T. D., and C. N. YANG: Phys. Rev. 87, 410 (1952).

11. Ahlfons, L. A.: Complex analysis, p. 222. New York: McGraw-Hill 1966.

12. Gallavotiti, G., S. Mrracle-Sole, and D. W. Robinson: Phys. Letters 25 A, 493 (1967); R. L. Dobrushin et al., to be published.

13. YANG, C. N., and T. D. LeE: Phys. Rev. 87, 404 (1952).

${ }^{4}$ In the modified distribution functions and Ursell functions, defined in [23], the various arguments are allowed to represent the same particle.

9 Commun. math. Phys., Vol. 11 
14. Titchmarsh, E. C.: Theory of functions, p. 168. Oxford: University Press 1939.

15. BAKer, G. A., JR.: Phys. Rev. 161, 434 (1967).

16. Ahlfors, L.: Complex analysis, p. 176. New York: McGraw-Hill 1966.

17. Groenneveld, J.: Eq. (53) on p. 244 of Graph theory and theoretical physics (Proceedings of the NATO Summer School, Frascati, Italy, 1964; editor F. Harary). New York: Academic Press 1968.

18. Uhlenbeck, G. E., and G. W. Ford: Studies in statistical mechanics, Vol. 1, p. 143, equations (47) and (49). Amsterdam: North-Holland Publishing Co. 1962.

19. Griffiths, R. B.: J. Math. Phys. 8, 484 (1967).

20. Kelly, D. G., and S. Sherman: J. Math. Phys. 9, 466 (1968).

21. Elvey, J. S. N., and O. Penrose: Phys. Letters 26 A, 456 (1968).

22. Fisher, M. E.: J. Math. Phys. 6, 1643 (1965).

23. Lebowitz, J. L., and J. K. Percus: J. Math. Phys. 4, 116 (1963).

24. Gallavotti, G., and S. Miracle-Sole: Commun. Math. Phys. 7, 274 (1968).

O. Penrose

Department of Mathematics, Imperial College London S.W. 7, Great Britain 